\title{
Chronic abdominal pain in children: the importance of endoscopy
}

Children with intermittent or constant abdominal pain are common and can pose significant concerns to their parents and guardians [1]. In most cases, children with abdominal pain require only a good history, physical examination and some simple laboratory tests to rule out specific causes. If detailed history, physical examinations and simple laboratory tests fail to suggest any specific causes, the abdominal pain is not serious and the children will get better without treatment [1]. However, a significant proportion of children have chronic abdominal pain that lasts a long time or happens over and over again without any clues from history, physical examinations, and common tests [1]. Constant search for alarm findings will be needed in these children.

Some alarm findings warrant further investigations [2-5], such as involuntary weight loss, persistent abdominal pain and vomiting, gastrointestinal bleeding, chronic diarrhea, unexplained fever, urinary abnormalities, family history of peptic ulcer disease, oral aphthous ulcers, and localized abdominal pain. Alarm findings suggestive of Helicobacter pylori infection include upper abdominal or epigastric pain or discomfort with or without vomiting and refractory iron deficiency anemia.

When a suspicion for H. pylori infection is entertained, physicians seek to obtain a definitive diagnosis using either invasive [6] or noninvasive technique [7]. During endoscopy, the definitive diagnosis of $H$. pylori infection is done by one of three methods: biopsy urease test, histology, and much less commonly by bacterial culture. Noninvasive tests for the diagnosis of $H$. pylori include urea breath testing (UBT), stool antigen testing, and serology. UBT is based on an indirect test comparison with potential for bias due to confounding. The thresholds used for noninvasive tests were highly variable to be useful in clinical practice.
The sensitivity of biopsy urease testing is high [6]. This diagnostic technique is less expensive than histology in confirming the presence of $H$. pylori infection. Furthermore, the tissue biopsies can be used for rapid urease test. The study by Niyasom et al. suggests that the presence of antral nodularity has a high specificity for $\mathrm{H}$. pylori infection and can be used to guide treatment with proton pump inhibitors, amoxicillin, and clarithromycin with $100 \%$ improvement in abdominal pain within 4 weeks after treatment [8].

However, the consensus group of the European Society for Paediatric Gastroenterology Hepatology and Nutrition/North American Society for Pediatric Gastroenterology recommended that invasive diagnostic testing for $H$. pylori be performed only when treatment will be offered if tests are positive [9]. Therefore, endoscopic study will be advisable when the alarming symptoms are present (upper abdominal/epigastric pain plus persistent anemia), and treatment will be offered if the findings are positive for $H$. pylori infection guided by the presence of antral nodularity and biopsy or rapid urease testing.

As recommended by the European and North American consensus group, to reach the aim of a $90 \%$ eradication rate with initial therapy, antibiotics should be tailored according to susceptibility testing. This would require the result of bacterial culture and sensitivity test to tailor antibiotic regimens accordingly [9].

Therefore, endoscopic investigation of chronic abdominal pain in children may be appropriate given the presence of alarm findings. However, whether we need to wait for the result of bacterial culture and susceptibility testing is not completely answered. The risk and benefit of waiting for the result of bacterial culture and sensitivity test warrant further investigations to optimize cure rate and prevent unwarranted inappropriate use of antibiotics.

*Correspondence to: Editorial Office of Asian Biomedicine, Faculty of Medicine, Chulalongkorn University, Bangkok 10330, Thailand, e-mail: abmjournal@chula.ac.th

O Open Access. ๑ 2019 Editorial Office of Asian Biomedicine, published by Sciendo. (๔) BY-NC-ND This work is licensed under the Creative Commons Attribution NonCommercial-NoDerivatives 4.0 License. 
Since most chronic pain in children is functional in nature, the first goal of treatment is to help the child return to normal activities such as schooling and playing with other children. It may take time to find the causes of the pain and its treatment. Therefore, health providers and family members must help the child cope with pain via building good relationship between providers, parents and the children. This will render providers to manage the pain either functional or organic by offering sympathy, support and reassurance and avoid reinforcing the pain by giving it undue attention.

\section{References}

[1] Landry BW, Fischer PR, Driscoll SW, Koch KM, Harbeck-Weber C, Mack KJ, et al. Managing chronic pain in children and adolescents: a clinical review. PM R. 2015; 7(11 Suppl):S295-315. doi:10.1016/j. pmrj.2015.09.006.

[2] American Academy of Pediatrics Subcommittee on Chronic Abdominal Pain. Chronic abdominal pain in children. Pediatrics. $2005 ; 115: 812$.
[3] Chiou E, Nurko S. Functional abdominal pain and irritable bowel syndrome in children and adolescents. Therapy. 2011; 8:315.

[4] Noe JD, Li BU. Navigating recurrent abdominal pain through clinical clues, red flags, and initial testing. Pediatr Ann. 2009; 38:259.

[5] Rasquin A, Di Lorenzo C, Forbes D, Guiraldes E, Hyams JS, Staiano A, Walker LS. Childhood functional gastrointestinal disorders: child/adolescent. Gastroenterology. 2016; 150:1456.

[6] Chey WD, Wong BC, Practice Parameters Committee of the American College of Gastroenterology. American College of Gastroenterology guideline on the management of Helicobacter pylori infection. Am J Gastroenterol. 2007; 102:1808.

[7] Best LM, Takwoingi Y, Siddique S, Selladurai A, Gandhi A, Low B, et al. Non-invasive diagnostic tests for Helicobacter pylori infection. Cochrane Database Syst Rev. 2018; 3:CD012080. doi:10.1002/14651858.CD012080.pub2.

[8] Niyasom C, Ngoenmak T, Uthaisangsook S. Endoscopic finding and treatment outcome of children with Helicobacter pylori. Asian Biomed (Res Rev News). 2019; 13: xx-xx.

[9] Jones NL, Koletzko S, Goodman K, Bontems P, Cadranel S, Casswall T, ESPGHAN, NASPGHAN. Joint ESPGHAN/NASPGHAN Guidelines for the management of Helicobacter pylori in children and adolescents (update 2016). J Pediatr Gastroenterol Nutr. 2017; 64:991-1003. doi:10.1097/MPG.0000000000001594. 